\title{
Effect of music and toys on reducing pain during colonoscopy and acceptance of colonoscopy by children: a randomized clinical trial
}

\author{
Mohammad Ali Kiani ${ }^{1}$, Farhad Heydarian ${ }^{1}$, Zahra Feyzabadi ${ }^{1}$, Masumeh Saeidi ${ }^{2}$, Seyed Ali Jafari ${ }^{1}$, Paria \\ Hebrani $^{3}$
}

${ }^{1}$ MD, Associate Professor of Pediatrics, Department of Pediatrics, Faculty of Medicine, Mashhad University of Medical Sciences, Mashhad, Iran

${ }^{2}$ Department of Medical Education, Faculty of Medicine, Tehran University of Medical Sciences, Tehran, Iran

${ }^{3}$ Psychiatry and Behavioral Sciences Research Center, Mashhad University of Medical Sciences, Mashhad, Iran

\section{Type of article: Original}

\begin{abstract}
Background: Pain is an unpleasant sensory and emotional experience accompanied by fear and anxiety. Pain anxiety can cause resistance, reduce his/her cooperation and cause undesirable mental and psychological complications. The use of non-medical methods in relieving pain is preferable.

Objective: The purpose of this study was to determine the effectiveness of toys and music on pain and vital signs of children during colonoscopy.

Methods: This clinical trial was conducted on 90 children aged 2-14 years old referred to Ghaem Hospital in Mashhad (Iran) in 2016-2017. Performing colonoscopy on children who were randomly allocated to three equal groups of 30 (Two intervention and one control group): Group 1: toys (a car for boys and a doll for girls), Group 2: a piece of Clayderman music, and a control group. The vital signs of the children before and after colonoscopy, and the pain after it, were measured. Data were analyzed by SPSS version 16, using paired-samples t-test. A pvalue of less than or equal to 0.05 was considered statistically significant for examining the hypotheses of the study.

Results: The mean age of children was $9.07 \pm 3.22$, and $54.4 \%$ of them were girls. Heart rate, systolic and diastolic blood pressure increased significantly after colonoscopy in the music and control groups $(\mathrm{p}<0.001)$. However, in the toy group, mean heart rate, systolic and diastolic blood pressure decreased after intervention $(\mathrm{p}=0.147, \mathrm{p}=0.213, \mathrm{p}=0.246$ respectively). The mean score of pain in the toy group was significantly lower than the other two groups $(\mathrm{p}<0.001)$.

Conclusion: The results of this study showed that the use of toys and music were effective in reducing pain and accepting colonoscopy for the children, although the positive effects of the toy were more evident on the children's vital signs and pain. Therefore, the use of non-medication interventions can be considered as an effective way to accept colonoscopy and reduce pain in children.

Clinical Trial Registration: This trial is registered on the Thai Clinical Trials Registry, with the ID: TCTR20180507001

Funding: This project was funded by the research deputy of Mashhad University of Medical Sciences, (Mashhad, Iran).

Keywords: Children, Colonoscopy, Music, Pain, Toy, Vital signs
\end{abstract}

Abbreviations / Acronyms:

CONSORT: Consolidated Standards of Reporting Trials; DoH: Declaration of Helsinki; FALCC Scale: Face, Legs, Activity, Cry, Consolability scale

\section{Corresponding author:}

Associate Professor Dr. Mohammad Ali Kiani, Department of Pediatrics, Faculty of Medicine, Mashhad University of Medical Sciences, Mashhad, Iran. Tel: +98-05138412069, Email: Kianima@mums.ac.ir

Received: April 25, 2019, Accepted: September 12, 2019, Published: December 2019

This article has been reviewed / commented by three experts

Ethics approval: Ethics Committee of Mashhad University of Medical Sciences (Ref: 930162).

(C) 2019 The Authors. This is an open access article under the terms of the Creative Commons Attribution-NonCommercialNoDerivs License, which permits use and distribution in any medium, provided the original work is properly cited, the use is non-commercial and no modifications or adaptations are made. 


\section{Introduction}

Pain is a complex phenomenon influencing people of all ages. The International Association for the Study of Pain describes pain as an unpleasant sensory and emotional experience associated with potential or actual tissue damage. Pain is influenced by the individual's previous experience of it. Pain in infants, children and adults is often less than estimated and then less treated. Given the importance of pain management in children, studies have shown that children who experience pain will have long-term changes in pain perception and related behaviors $(1,2)$. The American Academy of Children and the American Pain Board recommend the use of pain killers and pain relief methods in painful processes for children (3-5). The use of medication is less advisable due to their problems and complications, while the use of non-medication cognitive and supportive behaviors such as watching movies, listening to music, reading books, playing with and using toys, and the family presence when performing painful processes, massage, touching, warming and cooling the painful position have shown positive results in reducing the pain of children $(3,6,7)$. Colonoscopy is now used as a standard and valuable method for early detection of colorectal injuries and ulcers. This diagnostic and therapeutic tool is also used in children and infants to evaluate and treat diarrhea, weight loss, abdominal pain, anemia associated with iron deficiency and rectal bleeding. The main concern during this process, which lasts 20 to 30 minutes, is the pain and fear of colonoscopy, resulting in a lack of cooperation between the children (8-10). Several techniques, including the use of sedatives, nitric oxide, carbon dioxide, hypnosis, and music therapy have been used to reduce pain and anxiety during this procedure $(11,12)$. In a meta-analysis conducted by Tam et al., data analysis showed that listening to music during colonoscopy significantly reduces the time of conducting this process and reduces the amount of sedative used (13). In another meta-analysis conducted by Rudin et al., results showed that listening to music during endoscopy results in lower levels of anxiety, less need for sedatives and reduction in the total time of process (14). The effects of music therapy on the mind and body have been known for centuries and the use of music therapy is common in the management of pain in medical sciences as an auxiliary tool $(15,16)$. The Colonoscopy of the digestive system is a diagnostic and therapeutic method for digestive diseases in children. Due to their being sensitive with low threshold for pain tolerance, some methods should be found to reduce children's pain and anxiety while conducting this process. According to limited studies conducted in this area, in this study, it was attempted to investigate the effect of music and toys on children's pain and their vital signs during colonoscopy.

\section{Material and Methods}

\subsection{Research design and setting}

This clinical trial was conducted from June-September 2017 in a colonoscopy ward of Ghaem Hospital in Mashhad (Iran). This study was conducted on 90 children of two to fourteen years of age who were candidates of colonoscopy for any reason and whose colonoscopy was performed in Ghaem Hospital of Mashhad. Ghaem Hospital is one of the two educational hospitals in Mashhad affiliated to Mashhad University of Medical Sciences. This educational hospital receives patients from Mashhad and other patients referring from the North East and South East of Iran. Children were randomly attributed to three groups of 30 people (two study groups and one control group).

\subsection{Sample size}

In a study conducted in the endoscopy ward of Ghaem Hospital of Mashhad, sample size was determined considering the formula of sample size and pain variable in study and control groups to be 60 subjects for study (toy and music groups) and 30 subjects for control group with confidence level of $90 \%$ and power of $90 \%$. This method of determining sample size was conducted based on similar previous studies $(17,18)$.

\subsection{Sampling}

Non-purposeful simple sampling was used and the children were randomly allocated to three groups of 30 people (two intervention groups and one control group).

\subsection{Selection criteria}

\subsubsection{Inclusion criteria}

The following were set as the inclusion criteria of the study: 1) age between 2-14 years, 2) indications for colonoscopy including lower gastrointestinal bleeding, polyps, irritable bowel syndrome, and 3) willingness to participate in the study.

\subsubsection{Exclusion criteria}

Exclusion criteria were hearing disorder, not wanting to listen to music, occurrence of bleeding or complication during colonoscopy and being under two years of age. 


\subsection{Blinding and randomization}

In this single-blind clinical trial, children were randomly attributed to two study groups (music and toy) and one control group such that children referring on odd days were placed in the study group and those referring on even days were attributed to the control group. Face, Legs, Activity, Cry, Consolability scale (FALCC scale) and BakerWong pain checklists were filled for both groups before and after intervention by a technician and a nurse, who were not aware of the study and control groups. In addition, heart rate and blood pressure were measured by these personnel before and after intervention. Figure 1 illustrates the CONSORT 2010 flowchart of the study.

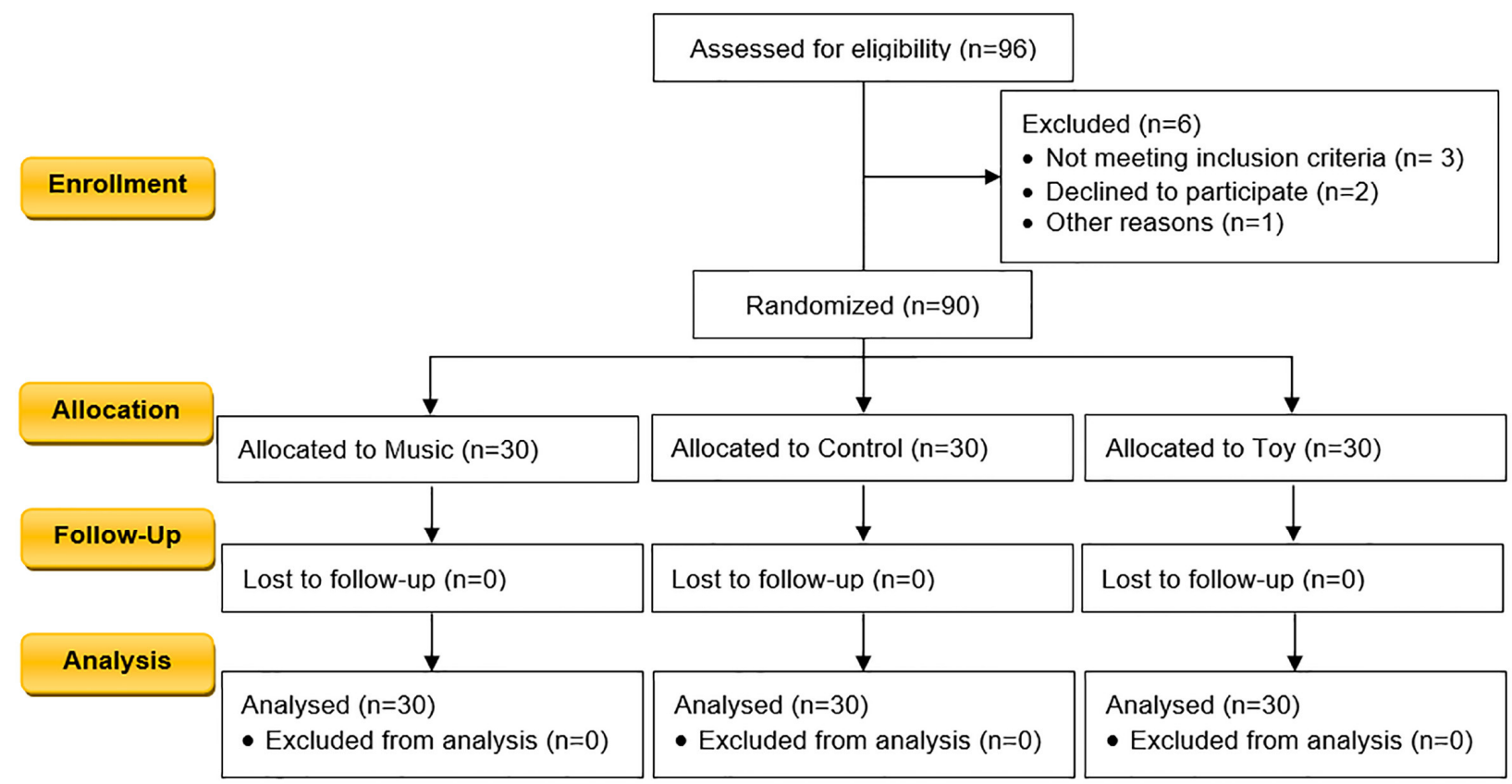

Figure 1. CONSORT*2010 Flow Diagram

* Consolidated Standards of Reporting Trials

\subsection{Outcomes}

The aim of this study was to determine the effect of music on pain reduction in children under colonoscopy. Another objective of this study was to measure heart rate and systolic and diastolic blood pressure in children of study and control group.

\subsection{Intervention}

In the study and control groups, $0.1 \mathrm{mg} / \mathrm{kg}$ midazolam with maximum dose of $3 \mathrm{mg}$ was prescribed for reducing patients' pain. For the study group, classical music by Clayderman was played during colonoscopy (from entering the colonoscopy room until the end of the process) using a digital $\mathrm{mp} 3$ player and two speakers; in the toy group, a standard toy for the child's age group (doll for girls and a car for boys) was considered from the time the child entered the colonoscopy room until the end of the colonoscopy process. The control group was subjected to colonoscopy procedures in accordance with colonoscopy standards without any intervention.

\subsection{Instrument}

After conducting colonoscopy, FALCC scale and Baker-Wong pain scale $(19,20)$ were filled by a technician and a nurse for both study and control groups. FALCC scale is a test composed of five factors of face, activity, legs, crying, and consolability, where the observer scores these factors based on the available condition between 0 and 20 . Baker-Wong pain scale is also a simplified tool to measure the amount of pain in patients where six smiley faces are shown to the patient and they are asked to select one face based on their state (Figure 2). This scale provides 6 photographic images of children with different degrees of pain, along with 0-5 scores. The scale shows a series of faces ranging from a happy face at 0 , which represents "no hurt" to a crying face at 10 which represents "hurts worst." Based on the faces and descriptions, the patient chooses the face that best describes their level of pain; this tool was originally created with children for children to help them communicate about their pain. Now the scale is 
used around the world for children, facilitating communication and improving assessment so that pain management can be addressed. At the end of the colonoscopy, the technician chose one of them based on the child's face image, indicating the amount of pain in the child. In addition, the child's vital signs, including heart rate and systolic and diastolic blood pressure, were measured before and after the procedure, for all children.

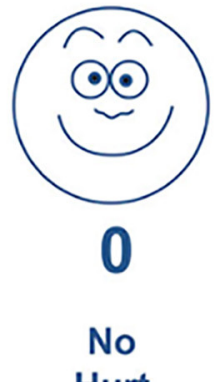

Hurt

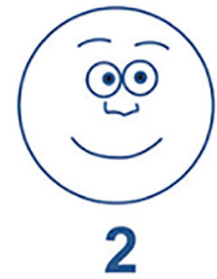

Hurts Little Bit

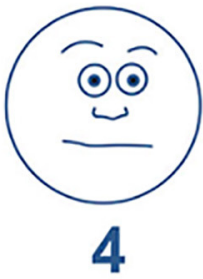

Hurts Little More

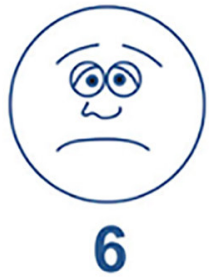

Hurts Even More

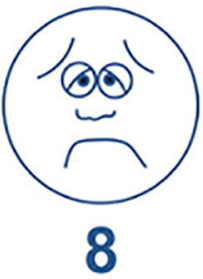

Hurts Whole Lot

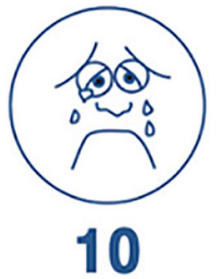

Hurts Worst

Figure 2. Wong Baker FACES Pain rating scale (19)

\subsection{Ethical considerations}

The Declaration of Helsinki (DoH) of ethical principles regarding human experimentation was considered fully during the study. This study was approved by the Ethics Committee of Mashhad University of Medical Sciences (Ref: 930162). Attending this study was voluntarily and participants were allowed to leave the study without any response whenever they wanted. When conducting this study, sufficient explanations were provided by authors for patients and their parents on the plan and the way of conducting it, and parents' written consent was obtained. Possible advantages and dangers were explained for patients and their parents. Furthermore, the way of compensating possible dangers were explained for them in detail. In addition, patients and their parents' possible questions were answered and one of the researchers' phone number (a pediatrician) was given to all participants of the study and this physician answered all questions of parents and patients. Moreover, when conducting the study, sample size was considered by researchers while respecting them and observing patients' rights as well as obtaining the trust of all participants.

\subsection{Statistical analysis}

Data were analyzed by SPSS software version 16.0 (SPSS Inc., Chicago, Illinois, USA). We used the mean and standard deviation to describe the quantitative variables and frequency, whereas percentages were used to describe the qualitative variables. The normality of the data distribution was examined using the Kolmogorov-Smirnov test. Paired-samples t-test was used to compare the mean difference in groups for data analysis and the ANCOVA with adjusted baseline values was used to compare the variables in three groups. A p-value of $\leqslant 0.05$ was considered as the significance level.

\section{Results}

Of the 90 children aged 2-14 years, $54.4 \%(\mathrm{n}=49)$ were girls. No significant difference was observed in the children under study in the study and control groups $(\mathrm{p}=0.17)$. The mean age of the children was $9.07 \pm 3.22$ years and this difference was not significant in both groups $(\mathrm{p}=0.218)$. Mean heart rate, systolic and diastolic blood pressure and pain in the study and control groups are shown in Table 1 before and after colonoscopy. Comparing mean heart rate before and after colonoscopy in both groups using Mann-Whitney test, showed that heart rate in the toy group was lower before and after colonoscopy compared to the control group ( $\mathrm{p}>0.05$ ). Results of the Mann-Whitney statistical test showed that heart rate before and after colonoscopy, was significantly increased in the music group compared to the toy group $(\mathrm{p}=0.001)$. Results of the Mann-Whitney statistical test showed that there was a significant difference in the music and control groups in terms of heart rate before and after endoscopy $(p=0.001, p=0.001)$. Comparing mean systolic blood pressure before and after colonoscopy in both groups using Mann-Whitney test, showed that systolic blood pressure in the toy group was lower before and after colonoscopy compared to the control group ( $>0.05)$. Results of the Mann-Whitney statistical test showed that systolic blood pressure before and after colonoscopy, was significantly increased in the music group compared to the toy group $(\mathrm{p}=0.001$ ). Results of the Mann-Whitney statistical test showed that there was a significant difference in the music and control groups in terms of systolic blood pressure before and after colonoscopy $(\mathrm{p}=0.001, \mathrm{p}=0.001)$. Comparing mean diastolic blood pressure before and after colonoscopy in both groups using Mann-Whitney test, showed that diastolic blood pressure 
in the toy group was lower before and after colonoscopy compared to the control group ( $\mathrm{p}>0.05$ ). Results of the Mann-Whitney statistical test showed that diastolic blood pressure before and after colonoscopy, was significantly increased in the music group compared to the toy group $(\mathrm{p}=0.001)$. In addition, the difference of diastolic blood pressure before and after colonoscopy showed a significant difference between the control and music groups $(p=0.001)$. In the present study, FALCC scale and Baker-Wong pain scale were used to evaluate pain in patients before, during and after colonoscopy. Results of the Baker-Wong scale showed that the difference in score of this test before and after colonoscopy was significantly lower in the study group compared to the control group $(p<0.000)$. On the other hand, average score of FALCC scale in both groups showed that the score of this test was significantly lower in the study group compared to the control group $(\mathrm{p}<0.000)$.

Table 1. A comparison of heart rate, systolic and diastolic blood pressure before and after colonoscopy for study and control groups

\begin{tabular}{|l|l|l|l|l|}
\hline Variables & Status & Toy group & Music group & Control group \\
\hline \multirow{5}{*}{ Heart beat } & Before Intervention & $132.93(26.93)$ & $78.63(13.85)$ & $100.33(11.45)$ \\
\cline { 2 - 5 } & After Intervention & $127.37(20.89)$ & $96.17(9.99)$ & $116.87(13.62)$ \\
\cline { 2 - 5 } & p-value & 0.147 & 0.001 & 0.001 \\
\hline Systolic blood pressure & Before Intervention & $113.60(16.11)$ & $90.33(7.98)$ & $96.07(9.67)$ \\
\cline { 2 - 5 } & After Intervention & $109.93(10.52)$ & $101.43(6.21)$ & $104.83(11.41)$ \\
\cline { 2 - 5 } & p-value & 0.213 & $<0.001$ & $<0.001$ \\
\hline \multirow{3}{*}{ Diastolic blood pressure } & Before Intervention & $74.10(9.57)$ & $55.83(5.74)$ & $63.17(9.42)$ \\
\cline { 2 - 5 } & After Intervention & $72.10(9.42)$ & $65.83(8.72)$ & $69.83(9.67)$ \\
\cline { 2 - 5 } & p-value & 0.246 & 0.001 & 001 \\
\hline Pain $(\mathrm{p}<0.001)^{*}$ & $4.33(0.55)$ & $4.93(0.25)$ & $5.00(0.00)$ \\
\hline
\end{tabular}

The data are presented as Mean (SD). *p-value

\section{Discussion}

In this clinical trial, colonoscopy results of 90 children were compared and evaluated in the form of two groups of study (60 children) and control (30 children) on the effect of music and toys on heart rate, blood pressure and pain in patients. Of these children, 49 subjects $(54.4 \%)$ were male and 41 subjects $(45.6 \%)$ were female. In terms of age, most children were between 8 and 12 years old (41.3\%), with (34.7\%) over 12 years old and (24\%) under 8 years old. This sample size seems to be appropriate compared to similar studies $(8,17)$. Age and gender were of normal distribution in the study and this complied with most studies conducted in this field $(11,21)$. Vital signs including heart rate, systolic and diastolic blood pressure of patients can act as an index to show patients' anxiety. In our study, a significant difference was observed in heart rate before and after colonoscopy in the music and control groups $(p<0.001)$; however, this difference was not significant in the toy group $(p>0.05)$. A result of a study showed the use of carbon dioxide $\left(\mathrm{CO}_{2}\right)$ insufflation for reducing post procedural discomfort and pain was effective compared to air, in children undergoing colonoscopy (22).

In terms of systolic blood pressure, there was a significant difference before and after colonoscopy and the difference between these values in the music and control groups $(p=0.001)$; also, diastolic blood pressure before colonoscopy showed a significant difference between the music and control groups $(p=0.001)$. In addition, the difference in diastolic blood pressure before and after colonoscopy was significantly lower in the toy group compared to the control group ( $\mathrm{p}>0.05)$. In a study conducted by Kiani et al. on the effect of music on vital signs, pain and anxiety, heart rate was $20 \mathrm{bpm}$ higher in the control group compared to the study group; however, no significant difference was observed in average arterial pressure in both groups (8). In the study by Kotwal et al., it was shown that music influenced systolic and diastolic blood pressure and the number of patients' breaths, and there had been a significant difference between study and control groups; while, no significant difference in heart rate was observed in both groups (21). In a similar study conducted by Tazakori et al. in Ardebil, results showed that no significant difference exists between study and control groups in terms of systolic and diastolic blood pressure in various intervals (23). In the present study, FALCC scale and Baker-Wong pain scale were used to evaluate pain in patients before, during and after colonoscopy. Results of the Baker-Wong scale showed that the difference in score of this test before and after colonoscopy was significantly lower in the study group compared to the control group $(p<0.000)$. On the other hand, average score of FALCC scale in both groups showed that the score of this test was significantly lower in study group compared to control group $(\mathrm{p}<0.000)$ such that the group that listened to music, experienced less pain compared to the control group. In their study, Harikumar et al. concluded that despite the fact 
that no difference was observed in pain scores of both groups, discomfort score, time of recovery and doses of midazolam taken, were significantly lower in the study group compared to the control group (24). The study by Kiani et al. (2013) also showed that music can significantly decrease pain and anxiety score in the study group compared to the control group (8). On the other hand, there are studies such as Good (1995), Tanabe (2001), Kwekkeboom (2003), and Richard (2007) that have not been able to prove the effect of music on pain reduction (2528).

Listening to music during a painful experience distracts attention from anxiety and has a therapeutic effect by promoting relaxation responses $(9,18)$. Findings of similar studies suggested that listening to music during colonoscopy can reduce the pain and anxiety of children (17). The use of music during endoscopy can also help in reducing pain and anxiety and stabilize children's vital signs (29). Music can also reduce the amount of sedatives needed to perform colonoscopy (30). Due to the limited research in this field, the use of music in adult colonoscopy also has similar results and results in more satisfaction (31), reduction of anxiety level in colonoscopy without sedation (32), and also reduces dosage of sedative drugs, anxiety, pain and dissatisfaction in patients (33). In addition, the use of mint oil as a non-medicinal intervention can increase satisfaction and reduce pain in children during colonoscopy (17). Given the importance of aggressive interventions such as colonoscopy in the diagnosis and treatment of diseases in children, adoption and use of interventions and strategies to manage children's pain during these processes should not be neglected.

\section{Study limitations}

One of the limitations of this study is that the assessment of pain after colonoscopy was done using the picture scale on the basis of a child's face. Given the age range of these children, the use of more complex scales was not possible in some of the samples. Also, this was a partially blinded study because we made efforts to limit bias from the assessor, and because of respect for ethical issues, the blinding of children was not possible.

\section{Conclusions}

The findings of this study showed that the use of toys and music as a non-medical intervention during colonoscopy was effective in reducing pain and accepting colonoscopy by the child. The positive effects of the toy were more evident on the children's vital signs and pain. Therefore, it is recommended to use non-medicinal interventions as effective ways to accept colonoscopy and reduce pain in children. Conducting a complementary study on efficiency of this method on child candidates for colonoscopy with a larger sample size and methodology of this study can be a valuable path for later studies in the future.

\section{Acknowledgments:}

This study is extracted from the thesis by Zahra Feyzabadi (Thesis number: 930162, Mashhad University of Medical Sciences). All patients and personnel of the colonoscopy ward of Ghaem Hospital are appreciated for assisting the author in conducting the present study.

\section{Conflict of Interest:}

There is no conflict of interest to be declared.

\section{Authors' contributions:}

All authors contributed to this project and article equally. All authors read and approved the final manuscript.

\section{References:}

1) Hermann $\mathrm{C}$, Hohmeister J, Demirakça $\mathrm{S}$, Zohsel K, Flor H. Long-term alteration of pain sensitivity in school-aged children with early pain experiences. Pain. 2006;125(3):278-85. PMID: 17011707. DOI: 10.1016/j.pain.2006.08.026

2) Victoria NC, Murphy AZ. Exposure to Early Life Pain: Long Term Consequences and Contributing Mechanisms. Curr Opin Behav Sci. 2016;7: 61-68. PMID: 27525299. PMCID: PMC4979223. DOI: 10.1016/j.cobeha.2015.11.015

3) Aydin D, Sahiner NC. Effects of music therapy and distraction cards on pain relief during phlebotomy in children. Applied Nursing Research. 2017;33: 164-8. PMID: 28096012. DOI: 10.1016/j.apnr.2016.11.011.

4) Srouji R, Ratnapalan S, Schneeweiss S. Pain in children: assessment and nonpharmacological management. International Journal of Pediatrics. 2010; 201:1-11. PMID: 20706640. PMCID: PMC2913812. DOI: http://dx.doi.org/10.1155/2010/474838. 
5) Lee GY, Yamada J, Shorkey A, Stevens B. Pediatric clinical practice guidelines for acute procedural pain: a systematic review. Pediatrics. 2014; 133(3):500-15. PMID: 24488733. DOI: 10.1542/peds.2013-2744.

6) Sahiner NC, Bal MD. The effects of three different distraction methods on pain and anxiety in children. Journal of Child Health Care. 2016;20(3): 277-85. PMID: 26040282. DOI: 10.1177/1367493515587062.

7) Van Der Heijden MJ, Araghi SO, Van Dijk M, Jeekel J, Hunink MM. The effects of perioperative music interventions in pediatric surgery: a systematic review and meta-analysis of randomized controlled trials. PLoS one. 2015;10(8):e0133608. PMID: 26247769. PMCID: PMC4527726. DOI: 10.1371/journal.pone.0133608.

8) Kiani MA, Khodadad A, Mohammadi S, Ghayour Mobarhan M, Saeidi M, Jafari SA, et al. Effect of peppermint on pediatrics' pain under endoscopic examination of the large bowel. J HerbMed Pharmacol. 2013; 2(2): 41-44.

9) Trevisani L, Zelante A, Sartori S. Colonoscopy, pain and fears: Is it an indissoluble trinomial? World journal of gastrointestinal endoscopy. 2014;6(6):227-33. PMID: 24932374. PMCID: PMC4055991. DOI: 10.4253/wjge.v6.i6.227.

10) Yoshioka S, Takedatsu H, Fukunaga S, Kuwaki K, Yamasaki H, Yamauchi R, et al. Study to determine guidelines for pediatric colonoscopy. World journal of gastroenterology. 2017;23(31): 5773-9. PMID: 28883703, PMCID:PMC5569292. DOI: 10.3748/wjg.v23.i31.5773.

11) Pishnamaz Nishaburi R, Hashemian nejad N. Children endoscopic and endoscopic survey of upper and lower gastrointestinal system in 12 years in 1375 Ghaem Hospital. Pediatric doctoral thesis. Faculty of Medicine, Mashhad University of Medical Sciences. 2006.

12) Walsh S. The pediatric colonoscopy. Gastroentrology nursing. 1995; 18(2): 57-61.

13) Tam WW, Wong EL, Twinn SF. Effect of music on procedure time and sedation during colonoscopy: a meta-analysis. World J Gastroenterol. 2008; 14(34): 5336-43. doi: 10.3748/wjg.14.5336. PMID: 18785289, PMCID: PMC2744067.

14) Rudin D, Kiss A, Wetz RV, Sottile VM. Music in the endoscopy suite: a meta-analysis of randomized controlled studies. Endoscopy. 2007; 39(6): 507-10. doi: 10.1055/s-2007-966362. PMID: 17554644.

15) Good M. A comparison of the effects of jaw relaxation and music on postoperative pain. Nurs Res. 1995; 44(1): 52-7. doi: 10.1097/00006199-199501000-00010. PMID: 7862546.

16) Triller N, Erzen D, Duh S, Petrinec Primozic M, Kosnik M. Music during bronchoscopic examination: the physiological effects. A randomized trial. Respiration. 2006; 73(1): 95-9. PMID: 16293960, doi: $10.1159 / 000089818$.

17) Kiani MA, Naghafi M, Jafari SA, Ghayour mobarhan M, Mohammadi Sh, Saeidi M, et al. Effects of Music on Pain, Anxiety and Vital Signs of Children during Colonoscopy. Life Science Journal. 2013; 10(12): 313.

18) Najaphi M, Motamed F, Kiani MA, Khakshour A, Saeidi M, Jafari SA, et al. Effect Supermint oil (peppermint oil) on children's pain during colonoscopy. Journal of North Khorasan University of Medical Sciences. 2013; 5(2):476.

19) Garra G, Singer AJ, Taira BR, Chohan J, Cardoz H, Chisena E, et al. Validation of the Wong-Baker FACES Pain Rating Scale in pediatric emergency department patients. Acad Emerg Med. 2010; 17(1): 504. doi: 10.1111/j.1553-2712.2009.00620.x. PMID: 20003121.

20) Hockenberry MJ, Wilson D. Pain Assessment and Management in Children. In Hockenberry, M.J., Wilson, D. (2015), Wong's nursing care of infants and children (p. 155). St. Louis, MO: Mosby, an imprint of Elsevier Inc.

21) Kotwal MR, Rinchhen CZ, Ringe VV. Stress reduction through listening to Indian classical music during gastroscopy. Diagn Ther Endosc. 1998; 4(4): 191-7. doi: 10.1155/DTE.4.191. PMID: 18493472, PMCID: PMC2362603.

22) Homan M, Mahkovic D, Orel R, Mamula P. Randomized, double-blind trial of $\mathrm{CO}_{2}$ versus air insufflation in children undergoing colonoscopy. Gastrointest Endosc. 2016;83(5):993-7. PMID: 26363332. doi: 10.1016/j.gie.2015.08.073.

23) Tazakori Z, Amani F, Karimollahi M. The Effect of Music Therapy on Patients' Blood Pressure in Endoscopy Unit in Bou-Ali hospital, Ardebil. Iranian Journal of Nursing and Midwifery Research. 2007; 12(1): 10-2.

24) Harikumar R, Raj M, Paul A, Harish K, Kumar SK, Sandesh K, et al. Listening to music decreases need for sedative medication during colonoscopy: a randomized, controlled trial. Indian J Gastroenterol. 2006; 25(1): 3-5. PMID: 16567885. 
25) Good M. A comparison of the effects of jaw relaxation and music on postoperative pain. Nurs Res. 1995; 44(1): 52-7. doi: 10.1097/00006199-199501000-00010. PMID: 7862546.

26) Tanabe P, Thomas R, Paice J, Spiller M, Marcantonio R. The effect of standard care, ibuprofen, and music on pain relief and patient satisfaction in adults with musculoskeletal trauma. J Emerg Nurs. 2001; 27(2): 124-31. doi: 10.1067/men.2001.114386. PMID: 11275859.

27) Kwekkeboom KL. Music versus distraction for procedural pain and anxiety in patients with cancer. Oncol Nurs Forum. 2003; 30(3): 433-40. doi: 10.1188/03.ONF.433-440. PMID: 12719743.

28) Richards T, Johnson J, Sparks A, Emerson H. The effect of music therapy on patients' perception and manifestation of pain, anxiety, and patient satisfaction. Medsurg Nurs. 2007; 16(1): 7-14. PMID: 17441624.

29) Sabzevari A, Kianifar H, Jafari SA, Saeidi M, Ahanchian H, Kiani MA, et al. The effect of music on pain and vital signs of children before and after endoscopy. Electronic physician. 2017;9(7):4801-5. PMID: 28894538. PMCID: PMC5586996. DOI: 10.19082/4801

30) Lee DW, Chan K-W, Poon C-M, Ko C-W, Chan K-H, Sin K-S, et al. Relaxation music decreases the dose of patient-controlled sedation during colonoscopy: a prospective randomized controlled trial. Gastrointestinal endoscopy. 2002;55(1):33-6. PMID: 11756911. DOI: 10.1067/mge.2002.120387

31) Bechtold ML, Perez RA, Puli SR, Marshall JB. Effect of music on patients undergoing outpatient colonoscopy. World J Gastroenterol. 2006; 12(45): 7309-12. PMID: 17143946. DOI: PMC4087488

32) Ko C-H, Chen Y-Y, Wu K-T, Wang S-C, Yang J-F, Lin Y-Y, et al. Effect of music on level of anxiety in patients undergoing colonoscopy without sedation. Journal of the Chinese Medical Association. 2017;80(3):154-60. PMID: 27889459. DOI: 10.1016/j.jcma.2016.08.010

33) Ovayolu N, Ucan O, Pehlivan S, Pehlivan Y, Buyukhatipoglu H, Savas MC, et al. Listening to Turkish classical music decreases patients' anxiety, pain, dissatisfaction and the dose of sedative and analgesic drugs during colonoscopy: a prospective randomized controlled trial. World journal of gastroenterology: WJG. 2006;12(46):7532-36. PMID: 17167846. PMCID: PMC4087603. 